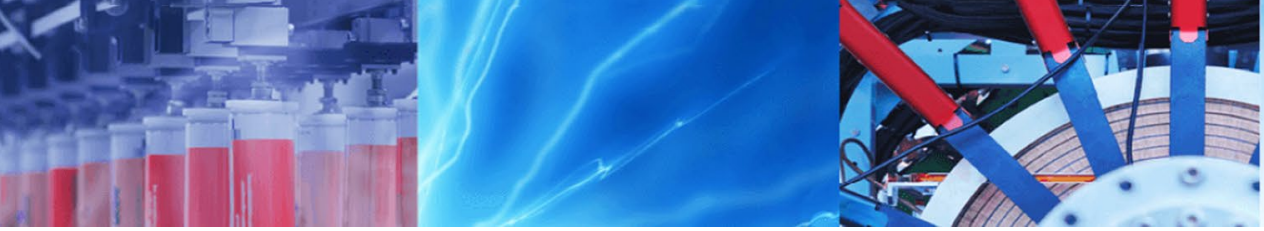

Research Article

\title{
Experimental investigation of electric discharge parameters in correlation with peak pressure at industrial electrohydraulic forming
}

\author{
I. Pérez ${ }^{1}$ (D) M. K. Knyazyev² ·J. San José
}

() Springer Nature Switzerland AG 2019

\begin{abstract}
The paper shows the investigation results of correlating electric discharge and pressure field parameters aimed to improvements in the electrohydraulic impact forming (EHF) technology at industrial application. The experimental research was performed by using a conical discharge chamber equipped with a set of two electrodes in semi-industrial EHF installation. Pressure fields along round flat area were measured by applying the multi-point membrane pressure gauge methodology. The conditions of the tests include a wide range of spark gaps with four levels of charge voltage and energy. Measurements of discharge voltage and current are performed with voltage divider, Rogovsky coil and electronic oscilloscope. Other electric discharge parameters were calculated from the recorded voltage and current curves. The essence of investigation is to analyse energy parameters for peak pressure of shock wave generated by these discharge energy parameters. Though these dependencies were earlier analysed theoretically and tested in electrohydraulic plants under laboratory conditions, the practical interest in this experimental investigation is to reveal influence of conditions of real semi-industrial EHF press designed for batch production of sheet parts. Conducted experimental investigation has shown that industrial applications of high-voltage non-initiated discharges can significantly deviate from the theoretical and laboratory results. Dependencies of peak pressure from maximum power during the first semiperiod of discharge current and slope of power curve appeared to be not so strong. These deviations in peak pressure can reach $20-30 \%$. Among the assumed additional factors influencing energy and pressure parameters are: condition of current-conductive rod of electrode (erosion, rust, radius); condition (wear) of electrode insulator (increase of naked area of current-conductive rod); variations in shape, position and length of discharge channel relative to spark gap; "shadowing" effect of electrodes at some positions of discharge channel relative to electrode; several discharge channels at small spark gap and other. To reveal effect of these factors the authors plan to carry-out tests with wire-initiated discharges to check the variations in shape, position and length of discharge channel relative to spark gap in the same discharge chamber configuration.
\end{abstract}

Keywords Electrohydraulic forming $\cdot$ Discharge parameters $\cdot$ Peal pressure $\cdot$ Voltage

\section{Introduction}

Electrohydraulic impact forming (EHF) is one of many methods of metalworking, that is, forming of metals with pressure forces. Therefore, pressure distribution along sheet blank surface plays a leading role in sheet blank deformation process.

Many factors influence pressure field parameters at non-initiated discharges: efficiency variations of energy evolving in a discharge channel, condition (wear) of work

\footnotetext{
$\triangle$ I. Pérez, inaki.perez@tecnalia.com; M. K. Knyazyev, m.knyazyev@khai.edu; J. San José, jsanjose@alfe.com | ${ }^{1}$ Industry and Transport Division, TECNALIA, Parque Tecnológico de Bizkaia, Astondo Bidea, Edificio 700, 48160 Derio, Spain. ${ }^{2}$ Department of Aircraft Engine Manufacturing Technologies, National Aerospace University, Chkalova St., 17, 61070 Kharkiv, Ukraine.
}

SN Applied Sciences (2019) 1:1299| https://doi.org/10.1007/s42452-019-1336-0

Received: 21 June 2019 / Accepted: 23 September 2019 / Published online: 28 September 2019 
surfaces of electrodes, variations in shape and position of discharge channel relative to electrodes, discharge chamber walls and a blank surface, etc.

When configuration of discharge chamber and position of single pair of electrodes relative to each other (spark gap, angular location), chamber's walls and sheet blank are fixed, it is assumed that main factors influencing pressure distribution are electric discharge parameters.

The Vohnout et al. [1] investigation, devoted to further illumination of the nature, origin and possible amelioration of the undesirable pressure heterogeneity at EHF, showed basic variability of the pressure distribution for fixed process parameters resulted from the 3-kJ discharges. Four titanium sheet samples demonstrated different levels of plastic deformation (pressure) on target grid holes including different quantities of the sheared holes out.

Homberg et al. [2] have conducted comparative investigations of sheet formability into $\mathrm{V}$-shape die with bottom radius $\mathrm{R}$ by static hydroforming and various impulse forming methods. Specimen produced by EHF method showed general dependency of smaller radii $\mathrm{R}$ (larger depth of groove shape) with the discharge energy enlargement. This dependency appeared to be not smooth and also the $\mathrm{R}$ deviations along the groove bottom were noticed that indicated instability of peak pressure generation even under laboratory conditions.

San José et al. [3] showed strong influence of electric parameters and spark gap of discharge circuit on peak pressure value and repeatability of pressure fields. For the specified geometric parameters of discharge chamber at satisfactory level of repeatability the following electric parameters were recommended: range of spark gap 17 to $22 \mathrm{~mm}$, voltage range 20 to $30 \mathrm{kV}$ and charge energy range 6.64 to $14.94 \mathrm{~kJ}$. The recommended parameters also generate the largest peak pressures of shock wave front. The experimental setup and results of that investigation served as a basis for this investigation with aim of more detailed analysis of influence of electric discharge parameters on shock wave peak pressure values.

San José et al. [4] have conducted the experimental forming of the part "lamp reflector" showed instability of the part geometric shape at the same test conditions including electric parameters of discharge circuit. Investigation of pressure field stability was conducted with varying spark gap / at the constant electric parameters: voltage $V=15 \mathrm{kV}$, capacitance $C=33.2 \mu \mathrm{F}$, charged energy $E=3.73 \mathrm{~kJ}$. Tests showed in general increasing a peak pressure of shock wave with spark gap increase. Investigators also revealed continuous decrease of discharges efficiency because of electrodes work surfaces erosion, increase of spark gap because of erosion, wear of positive electrode insulator and, crease of naked surface area of positive electrode.
Electric breakdown and discharge process in a liquid is a very complicated phenomenon. Experimental investigations of electric and energy discharge parameters were performed with aid of measurements of discharge current and voltage with oscilloscopes. The vast theoretical and laboratory investigations of electric and energy discharge parameters in correlation with shock wave peak pressure had been conducted in previous times (during the first wave of interest to EHF technology) and submitted in the book of Guly [5] and with deeper analysis of the correlation in the book by Krivitsky and Shamko [6]. Therefore, the following review information contains mainly fragments of the book by Krivitsky and Shamko [6] with fragments from other literature sources to complete this analytical review.

Representative oscillograms of discharge current $i$ and voltage $V$ are shown in Fig. 1. Changes in discharge modes from the oscillating to the aperiodic are made by increasing the discharge gap length / [6]. These discharges were initiated by aluminium wire insertion of very small diameter $3 \mu \mathrm{m}$ for stability of breakdown, discharge channel position and its configuration.

It is obvious from the oscillograms that the largest amplitude of current and the shortest duration of the first current semi-period are in the discharges with the shortest spark gap. The duration and amplitude of the first semiwave of discharge current $\tau_{1}$ values are practically equal to those of short-circuit discharge $(\pi \sqrt{L C})$.

Resistance of discharge channel during the first semiperiod goes down to some minimum value, then it increases and in the current node $(i=0)$ reaches the maximum, which is displaced, as a rule, into the second semiperiod. Minimum value of resistance is near to the current

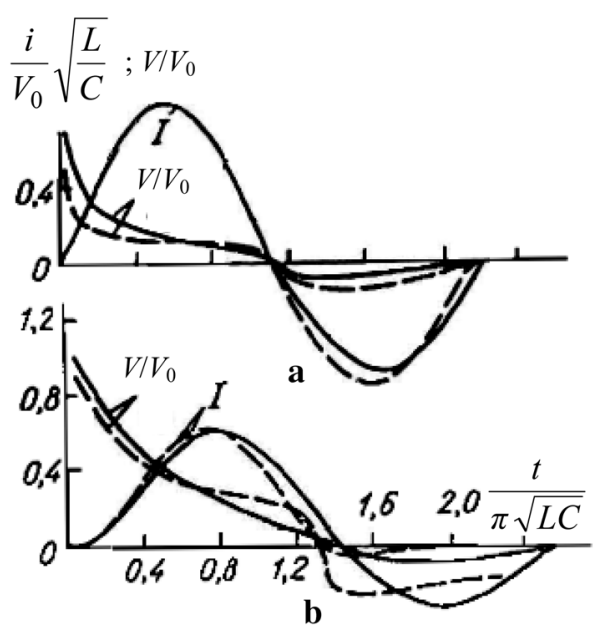

Fig. 1 Transformation of electric parameters curves when moving to long discharges: $\mathrm{a}$-solid lines: $V 0=40 \mathrm{kV}, C=0.15 \mu \mathrm{F}, L=0.4 \mu \mathrm{H}$, $I=20 \mathrm{~mm}$; dashed lines: $V O=14 \mathrm{kV}, C=99 \mu \mathrm{F}, L=10 \mu \mathrm{H}, I=55 \mathrm{~mm}$; b-solid lines: $V O=40 \mathrm{kV}, C=3.2 \mu \mathrm{F}, L=2.2 \mu \mathrm{H}, I=120 \mathrm{~mm}$; dashed lines: $V O=14 \mathrm{kV}, C=99 \mu \mathrm{F}, L=10 \mu \mathrm{H}, I=135 \mathrm{~mm}[6]$ 
maximum along time axis, though it also displaces to the larger times.

Resistance of discharge channel increases with increase of spark gap value.

In order to reveal and analyse the identical discharges their electric parameters were normalised to obtain dimensionless forms. Normalising factors are: for current-the reciprocal value of maximum possible current amplitude $V_{0} / \sqrt{L / C}$; for resistance-the reciprocal value of characteristic impedance $\sqrt{L / C}$; for time-the natural frequency value determined by duration of current semiperiod in the short-circuit mode $\pi \sqrt{L C}$.

Dimensionless diagrams of current and voltage (Fig. 1) were used for calculations of dimensionless curves of resistance.

In Fig. 1 the solid lines of current and voltage are for relatively short discharges at small capacitance $C$ with characteristic time $\pi \sqrt{L C} \leq 10 \mu$ s; dashed lines-for long discharges with $\pi \sqrt{L C} \leq 100 \mu \mathrm{s}$.

The longer discharges $\left(\tau_{1} \leq 100 \mu \mathrm{s}\right)$ differ by faster drop of voltage and resistance during the first quarter of current wave (dashed lines in Fig. 1). The current curves of long discharges are nearer to sinusoidal form than the short ones. Resistance of discharge channel reaches the minimal value much faster that appears smaller than the characteristic impedance $\sqrt{L / C}$.

Increase of spark gap / results in smaller slope of voltage curves during entire first current semi-period for discharges near to aperiodic (Fig. 1b). The oscillating discharges with smaller spark gaps (Fig. 1a) have faster drop of voltage and the voltage curve goes nearly parallel to the time axis along the first current semi-period.

Duration of the first semi-period of current with equal normalised current amplitude changes not equally. Thus, for oscillating discharges duration of the first current pulse $\tau_{1}$ practically does not differ from short-circuit duration $\pi \sqrt{L C}$ (Fig. 1a). For discharges near to aperiodic the difference can be significant-up to $0.15 \pi \sqrt{L C}$ (Fig. 1b).

Based on the theory of dimension and similarity, $[5,6]$ described transition process in discharge circuit in the normalised parameters. It allows determining the laws of all electric parameters during discharge depending on initial electric values.

One of the main similarity criteria is relative (normalised) portion of energy evolved during the first semiperiod of discharge current $\eta=E_{1} / E_{0}$, where $E_{0}$ is initial charged energy, $E_{0}=C V_{0}^{2} / 2$. This criterion is used for classification of discharges types and for determination of extreme cases in discharge of capacitance bank.

Transition process in discharge circuit is described by series of current and voltage curves (Fig. 2) for discrete values $\eta=0 \ldots 1$. The graphs show that transition process in discharge circuit with active resistance in the form of

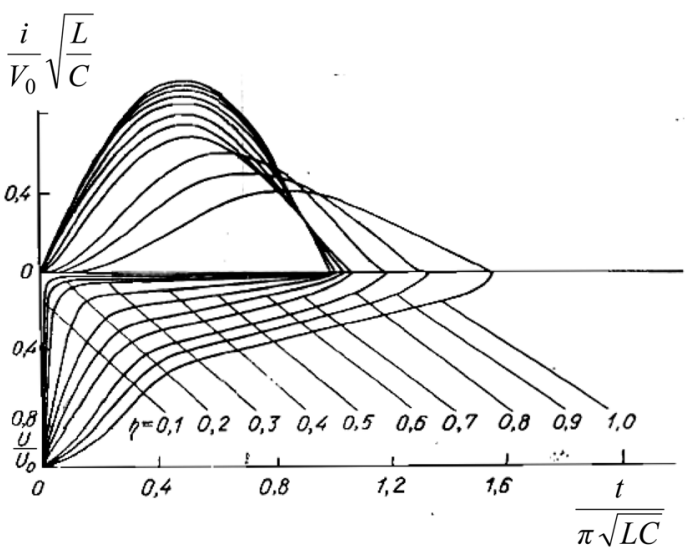

Fig. 2 Normalised curves of current and voltage for long discharges $\tau_{1}>40 \mu \mathrm{s}[6]$

discharge channel significantly differ from transition process with discharge of capacitance in inductance and constant active resistance even for oscillating modes. Slope of current curve in the linear circuit practically does not change and at the start moment $(t=0)$ equals $V_{0} / L$, when moving from oscillating mode to aperiodic one. In the case of considered nonlinear circuit, the slope of current curve can change tenfold and more. In addition, maximum of slope displaces from the start of discharge to the side of current amplitude.

It is impossible to characterise discharge by some angular frequency and approximating function of current by ordinary decadent sinusoid, because duration of the first semi-wave of discharge current can 1.5 and more times exceed the $\pi \sqrt{L C}$ value and duration of the following semi-waves.

Abrupt decrease (drop) of voltage is noticed in oscillating discharges with small $\eta$ values, when current amplitude is near to the $V_{0} \sqrt{C / L}$ normalising coefficient.

Pressure and temperature in the channel of impulse discharge in water, parameters of generated pressure pulses are caused by high-rate energy evolvement. Therefore, for practical application it is necessary to know the law of energy liberation, which, in its turn, is determined by external adjustable parameters of discharge circuit and spark gap value.

Maximal effect from underwater impulse discharge depends on both the energy in pressure pulse and the peak pressure at shock wave front. Parameters of pressure pulse can be expressed through energetic characteristics.

Energetic characteristics can be obtained from the solution of differential equation of discharge circuit with use of theory of dimension and similarity, as well as from systematic analysis of electric discharge parameters, application of empirical and semi-empirical dependencies for discharge current curves with time characteristic. 

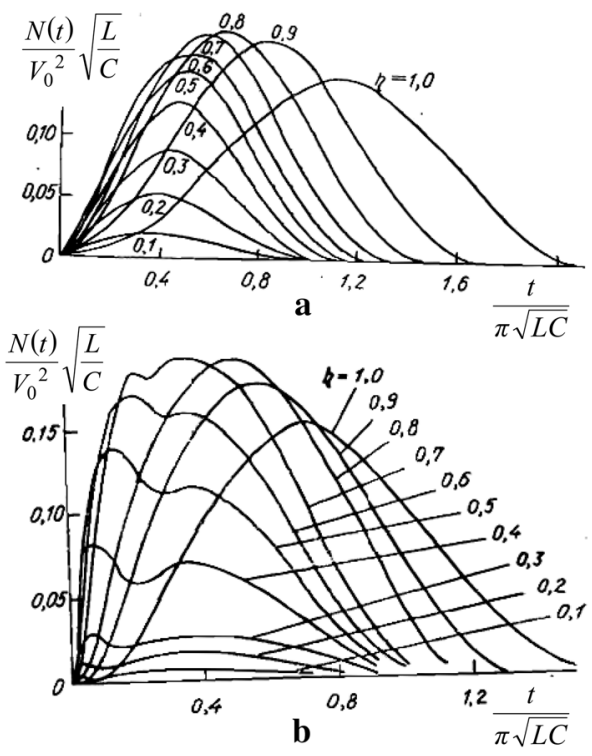

Fig. 3 Normalised curves of electric power for short $\tau_{1}<40 \mu \mathrm{s}$ (a) and long discharges $\tau_{1}>40 \mu \mathrm{s}$ (b) [6]

Changes in the speed of energy evolvement can be presented as series of normalised curves of electric power corresponding to the discrete values of criterion $\eta=0 \ldots 1$ (Fig. 3). Valuation was performed to the power $N_{0}$ value that equals a product of maximal possible amplitude of discharge current and maximal possible value of voltage, that is, charge voltage of capacitor bank

$N_{0}=V_{0}^{2} \sqrt{C / L}$

For short discharges (Fig. 3a) the slope of power impulse front is the largest and approximately equal for narrow range of discharge modes $0.6 \leq \eta \leq 0.8$

$\frac{d N}{d t}=(1.4-1.5) \frac{V_{0}^{2}}{L}$.

Amplitude values of power for discharges with $\eta=0.7-0.9$ practically coincide, but time $\tau_{N}$ for developing this maximum power is different.

For the oscillating modes with $\eta=0.1-0.3$ the start slope of power curve is much smaller than maximum possible value $\left(0.15 V_{0}^{2} / L\right)$, when comparing with respective characteristics of current curves.

Curves of normalised power for long discharges (Fig. 3b) significantly deviate from the curves of short discharges. It is explained by shift of the channel resistance drop to the beginning of discharge for the most of modes.

Increase in slope of power curves is observed for discharge modes with $\eta=0.1-0.7$ $\frac{d N}{d t} \approx 3 \frac{V_{0}^{2}}{L}$.

Characteristic feature of long-type discharges is presence of two maximums; one of them corresponds to the moment of current amplitude. Maximum power values for discharge modes with $\eta=0.7-0.9$ are practically coincide and equal to $\left(0.2 V_{0}^{2} \sqrt{C / L}\right)$. The modes with small value of the $\eta$ criterion have significant asymmetry of power pulse and shift of maximum curve slope to the discharge start.

During electric power rise to the $N_{\max }$ value in the time interval from 0 to $\tau_{N}$ the formation of front segment of shock wave occurs.

Results of experimental investigations confirmed that the front of shock wave departs from discharge channel at the beginning stage of its development, during the first semiperiod of current and electric power. Further portions of energy, evolved during the second and other semi-periods, do increase neither peak pressure value nor duration of shock wave. Hence, the mode of discharge determines efficiency of electric energy transformation into the pressure energy.

Dependency of amplitude power values from the length of spark gap has extremum nature that gives opportunity to determine optimal spark gap value by energetic parameters.

Power curve can be approximated by linear function $N=\gamma_{1} \cdot t$ that is performed only for critical modes of discharge or for limited time intervals $t<\tau_{N}$. Naugolnykh and Roy [7] proposed to approximate speed of energy evolvement (experimental power curve) by a triangle equal by area under the curve and, thus, providing more accurate determination of power slope $\gamma_{1}$ (Fig. 4).

During the tests performance [6] it was noticed that the peak pressure dependency changes the sign of its derivative $\frac{d}{d l} P_{m}(I)$, when modes of discharge were transformed from oscillating to critical by respective change of spark gap value I. Analysis of previous results shows that the same behaviour

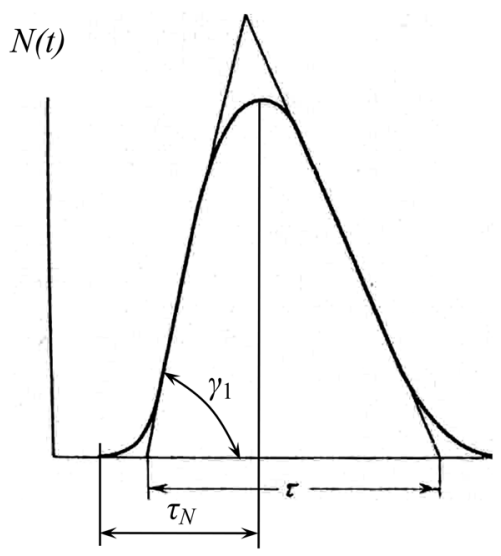

Fig. 4 Diagram for approximation of electric power curve [7] 
belongs to dependencies of power $N_{\text {max }}$ power per length unit of discharge channel $N_{\text {max }}^{0}$ slope of power curve $\gamma_{1}$, and slope of power curve per length unit of discharge channel $\gamma_{1} / l$. Therefore, it was assumed that

$P_{m} \sim f(r / I) F_{N^{\prime}}^{\delta}$

where $r$-coordinate of point relative to the discharge channel axis in meridional plane; $\delta$-constant determined by discharge conditions; $F_{N}$-one of the parameters listed above $\left(N_{\text {max }}, N_{\text {max }}^{0} \gamma_{1}, \gamma_{1} / l\right)$.

Based on the experimental data and approximation dependencies for plasma conductivity, radius of discharge channel and its volt-ampere characteristic the calculations of pressure in channel $P_{a}$ were performed [6]. Their results are submitted in the Fig. 5 as series of curves. Analysis of curves shows that value of discharge channel pressure follows the time dependency of electric power (Fig. 3a) in a qualitative aspect. The pressure increases, reaches the maximum value now near to the maximum power and decreases 1.5-2 times to the end of the first semi-period. It is noticeable that the dependency $P_{a}^{\max }(\eta)$ is rather weak and for rough calculations it can be ignored, assuming that $P_{a}^{\max } \sim\left(\rho V_{0}^{2} / L I\right)^{1 / 2}(\rho$ is a density of transmitting medium (water)).

Analysis of experimental and calculation results proved that the main factor influencing the value and form of pressure wave is slope $\gamma_{1}$ of electric power curve. For high-power discharges with inductance $L \sim 1 \mu \mathrm{H}(\tau \sim 100 \mu \mathrm{s})$ the power slope is $\gamma_{1} \sim 10^{14} \mathrm{~W} / \mathrm{s}$ (power slope per length unit of discharge channel $\gamma_{1} / / \sim 10^{15} \mathrm{~W} /(\mathrm{ms})$ ), which are lower values as compared with middle-power discharges with $\tau \sim 10 \mu \mathrm{s}$. Due to this feature formation of shock wave for mighty and long discharges occurs, as a rule, at the distance $r \geq l$.

Based on the fundamental equation of energetic balance in discharge channel and semi-empirical relationships for maximum power $N_{\max }$ and its time $\tau_{N}$ the authors [6] derived the formula for calculation of maximum pressure in discharge channel $P_{a}^{\max }$ with relative error up to $15 \%$

$P_{a}^{\max } \approx 0.17 \sqrt{\frac{\rho V_{0}^{2}}{L I}}$

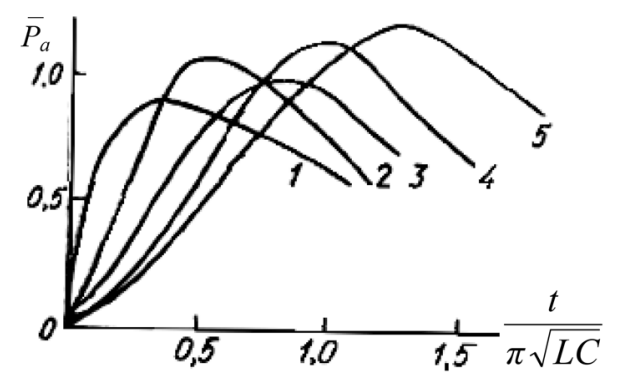

Fig. 5 Generalized dependencies of pressure in discharge channel: $1-\eta=0.3 ; 2-\eta=0.5 ; 3-\eta=0.7 ; 4-\eta=0.8 ; 5-\eta=0.9$ [6]
The formula (2) shows that maximum contribution for the $P_{a}^{\max }$ value the charged voltage $V_{0}$ does. The increase of voltage results in quasi-linear growth of the $P_{a}^{\max }$ value. The higher the medium density $\rho$, the higher the pressure in discharge channel is. Decrease of inductance $L$ and work spark gap / value causes the $P_{a}^{\max }$ growth. Capacitance nearly does not influence the pressure. However, increase in capacitance $C$ will result in increase of duration of the same pressure while the other conditions are equal.

Pressure of discharge channel is transmitted to the surrounding liquid during the process of its expansion in the form of shock wave.

Peak pressure of shock wave, when its cylindrical symmetry exists in the volume nearest to discharge channel at the distance $r<2.5 \mathrm{I}$, can be determined from

$P_{m}=\frac{b_{1}\left(\Pi_{k}\right)}{\sqrt{r}} \frac{\rho^{3 / 8} V_{0}^{5 / 4} C^{1 / 4}}{L^{3 / 8} / 5 / 8}$.

The tests showed that at the distance $2.5 I \leq r \leq 5.5$ I shock wave transforms from cylindrical to spherical. Peak pressure in this range

$P_{m}=\frac{1.33 b_{1}\left(\Pi_{k}\right)(10.1 r / l)}{\sqrt{r}} \frac{\rho^{3 / 8} V_{0}^{5 / 4} C^{1 / 4}}{L^{3 / 8} / 5 / 8}$

In the range of spherical symmetry of shock wave front at the distance $5.5 I<r<200 I$

$P_{m}=\frac{1.35 b_{1}\left(\Pi_{k}\right)}{r^{1.1}} \frac{\rho^{3 / 8} V_{0}^{5 / 4} C^{1 / 4}}{L^{3 / 8}}$

Here there are dimensionless function $b_{1}\left(\Pi_{k}\right) \approx 0.37 \Pi_{k}^{1 / 3}$ $e^{-12 \pi k}$ and similarity criterion

$\Pi_{k}=\frac{A l^{2}}{V_{0}^{2} \sqrt{L C}}$

Functions $P_{m}$ and $\Pi_{k}$ depend on spark gap / value, which, in its turn, influences resistance of discharge channel.

Analysis of above dependencies allows to make practically important conclusion. At the fixed distance $r$ there is an optimal value of spark gap $I$, which ensures generation of the largest pressure amplitudes $P_{m}$. In Fig. 6 the biggest peak pressure $P_{m}^{0}$ corresponds to optimal spark gap $I_{0}$.

For the most of practical tasks optimal spark gap value can be calculated from the formula derived from the function $P_{m}(l)$ for the distance $2.5 I \leq r \leq 5.5 /[6]$

$I_{0} \approx 0.3 \sqrt[4]{\frac{r^{2} z}{A}} E_{0}$ 


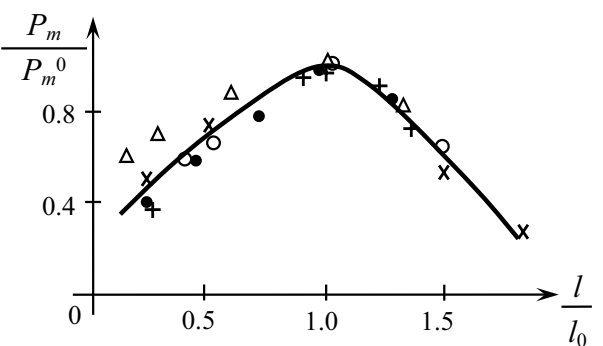

Fig. 6 Dependency of experimental values of peak pressure from spark gap value at $C=0.56 \mu \mathrm{F} ; V 0=40(O), 60(+)$ and $80(\bullet) \mathrm{kV}$; at $V 0=42.5 \mathrm{kV}(\Delta)$ and $C=3 \mu \mathrm{F}$; at $V 0=40 \mathrm{kV}, L=3 \mu \mathrm{H}(\times)$; solid line is the result of approximation of all experimental data [6]

where $z=\sqrt{L / C}$ is wave resistance of discharge circuit; $E_{0}=C V_{0}^{2} / 2$ is initial charged energy; $A$ is spark characteristic, for non-initiated discharges $A=10^{5} \mathrm{~V}^{2} \cdot \mathrm{s} / \mathrm{m}^{2}$.

This formula is obtained from the conditions of absorption of discharge energy by blank, equality of impulses of external and internal forces. Thus, selecting the optimal spark gap based on the fixed parameters $V_{0}, C, L, r$ one can generate shock wave with maximum pressure amplitude and, hence, the most effective mechanical work for low-inertia processes. Parameters $L, r$ should be selected as small as possible, considering the peculiarities of process and technological equipment.

All these investigation results are used by scientists and engineers, when developing the EHF equipment and processes, in designing of discharge chambers, organisation of discharge volume nearby of electrodes work ends, selection of electric parameters $(I, V, C)$ for discharges at different stages in sheet forming process. Usually inductance $L$ of discharge circuit is not changed (to be fixed possibly minimum for higher pressure). Also, the medium density $\rho$ is traditionally selected as water, though experimental investigations are conducted with different density media [8].

The above results [5-7] were obtained from the theoretical analysis of basic physical laws and "pure" laboratory tests.

Mamutov et al. [9] and Mamutov et al. [10] have performed the LS-DYNA simulation of discharge circuit and discharge channel as a source of impact pressure. Measurements of discharge current and voltage were performed with Rogovsky coil and divider for verification of simulation. Electric parameters provided long discharges with typical electric power curve calculated from the formula $N=i(t) \ldots U(t)$.

Ji-Yeon Shim and Bong-Yong Kang [11] have also performed the LS-DYNA simulation and measured discharge current at the EHF dome shape tests. They revealed enlargement of sheet sample deformation under shock wave pressure with increase of discharge current, charge voltage and energy.

Coman [12] in his experimental research confirmed linear dependency of peak pressure of shock wave from charge voltage that correlates with formula (2). He also obtained dependencies of peak pressure and spark gap like that shown in Fig. 6.

Operation of industrial EHF presses can deviate from those theoretical and laboratory conditions, because of design peculiarities of electrodes to withstand larger number of discharges, their relative position, presence of rigid walls of discharge chamber and electrodes nearby discharge volume, oxidation of contact surfaces in wiring of discharge circuit and many other factors not included into theoretical analysis.

The purpose of this work is to find correlation between theoretical results and their industrial realisation, to reveal some deviations, if any, in industrial machines.

In this work a research of electric discharge parameters with simultaneous measurements of peak pressure of shock wave was carried out. Tests were conducted in technological unit No. 2 of the semi-industrial installation electrohydraulic called UEHF-2, which is equipped with discharge chamber of conical shape with single electrode pair. Conical discharge chambers are rather typical for manufacture of small-size sheet components under smallbatch production conditions.

Methods of investigations are: measurements of discharge voltage and current with voltage divider and Rogowsky coil, processing the recorded curves and finding the characteristic energy parameters; pressure fields' measurements with multi-point membrane pressure gauge with 6-mm holes and aluminium membrane suitable for small-inertia processes and measurement of shock wave peak pressure.

\section{Experimental setup and measuring procedures}

In this work the used setup was like the work of San José et al. [3]. Tests have been carried with conical discharge chamber (Fig. 7) of $170 \mathrm{~mm}$ exhaust hole diameter. Loaded area was limited by spacer rings with internal diameters $D=150 \mathrm{~mm}$.

The clamping force of tooling pack was applied by a hydraulic cylinder of $60 \mathrm{kN}$ power. Holes in the discharge chamber adaptor were used for air evacuation and water supply (Fig. 7).

The intended spark gap value / was set up with threads performed in sleeves of electrodes and discharge chamber 


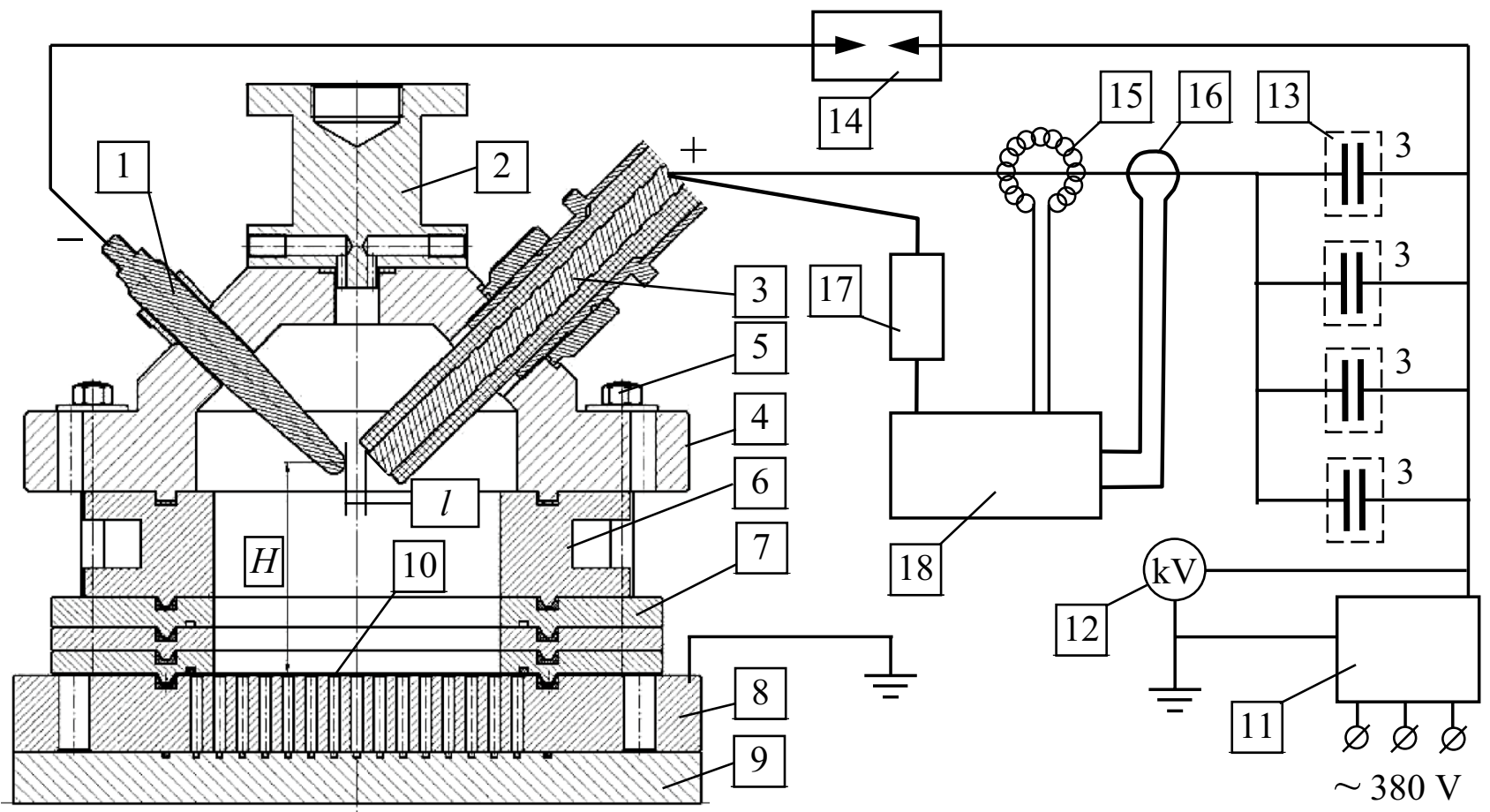

Fig. 7 Test diagram: 1-mass (negative) electrode; 2-upper adaptor; 3-insulated (positive) electrode; 4-discharge chamber; 5studs, nuts, washers; 6-spacer ring of $55 \mathrm{~mm}$ height; 7-spacer rings, hole diameter $150 \mathrm{~mm}$; 8 -membrane pressure gauge body; 9-lower adaptor; 10-membrane; 11-high-voltage charge unit;

holes. Distance $H$ between electrodes (discharge channel) and membrane 11 was approximately $110 \mathrm{~mm}$ with slight deviations when changing electrodes positions for spark gap setting, because electrodes axes are inclined.

During the tests performance the pulse generator provided the following electrical parameters: voltage $V_{0}=(10-30) \mathrm{kV}$, capacitance $C=33.2 \mu \mathrm{F}$, charged energy $E=(1.66-14.94) \mathrm{kJ}$.

The multi-point membrane pressure gauge (MPG) methodology [13] was applied to perform the pressure fields' measurements. To exclude the influence of hydraulic flow and pressure of vapour-gas bubble (low-inertia forming process) and to record only the pressure of shock wave, $t=1.0 \mathrm{~mm}$ thickness of membrane (A5052-O) and $d=6 \mathrm{~mm}$ diameter of holes in MPG body were selected.

To estimate the resulting action of the pressure of the shock wave the "equivalent static pressure" parameter was used, that is, by static pressure, which causes the same membrane deflection (plastic deformation) $h$. The Laplace's equation for spherical shell was used for calculating the equivalent static pressure (peak shock wave pressure)

$P=\frac{2 \sigma \cdot t}{R}$ 12-kilovoltmeter; 13-bank section of 3 capacitors; 14-discharge switch; 15-Rogowsky coil; 16-oscilloscope starting coil; 17-voltage divider; 18-oscilloscope; I-spark gap; $H$-distance between pressure gauge and discharge channel

where $t$-membrane thickness; $R$-radius of spherical segment, $R=\left(d^{2} / 4+h^{2}\right) / 2 h ; \sigma$-stress, at which deformation occurs.

Knyazyev and Zhovnovatuk [13] had noted that membrane gauges with geometric ratio $d / t<(6 \ldots 8)$ have linear proportionality between equivalent static pressure $P$ and peak pressure of shock wave $P_{m}$. Also, in the work of Antonenko et al. [14] based on experimental investigations it was shown that aluminium membrane gives "equivalent static pressure" approximately equal to peak pressure of shock wave $P_{m}$ in the gauge hole of $6 \mathrm{~mm}$ due to its low inertia.

In this work the average stress $\sigma$ for a spherical dimple was determined from the condition of equality of dimple material volumes before and after deformation.

In order to obtain relationship between impulse pressure and electric parameters the discharge curves of voltage and current were recorded with electronic oscilloscope PV65 (Fig. 7) and computer. Measurements of discharge voltage and current were arranged with voltage divider and Rogowsky coil produced and calibrated in the KhAl EHF laboratory. The starting coil was used to launch recording process.

Measurements results were recorded in the form of bmp pictures and data files with time step 50 and $100 \mathrm{~ns}$. 
Table 1 Tests conditions and processing results in average values

\begin{tabular}{|c|c|c|c|c|c|c|c|c|c|c|c|}
\hline \multicolumn{6}{|c|}{ Pressure tests [3] } & \multicolumn{6}{|c|}{ Electric parameters, average values } \\
\hline Series No. & $\mathrm{l}, \mathrm{mm}$ & $V_{0}, \mathrm{kV}$ & $E_{0}, \mathrm{~kJ}$ & $P_{m \text {-ave' }} \mathrm{MPa}$ & $k$ & $n$ & $N_{\text {max }}, \mathrm{MW}$ & $\gamma_{1}, \mathrm{MW} / \mu \mathrm{s}$ & $\eta$ & $\tau_{1}, \mu \mathrm{s}$ & $m$ \\
\hline 1. & 5 & 10 & 1.66 & 10.65 & 6 & 6.7 & 24.2 & 10.0 & 0.347 & 53.3 & 12 \\
\hline 2. & 5 & 15 & 3.73 & 29.04 & 3 & 7.5 & 38.1 & 5.1 & 0.225 & 59.8 & 6 \\
\hline 3. & 5 & 20 & 6.64 & 22.18 & 3 & 8.0 & 64.5 & 27.0 & 0.215 & 55.4 & 8 \\
\hline 4. & 5 & 30 & 14.94 & 57.71 & 5 & 8.5 & 153.5 & 57.9 & 0.177 & 52.3 & 8 \\
\hline 5. & 10 & 10 & 1.66 & 32.75 & 3 & 4.4 & 30.6 & 11.7 & 0.407 & 56.5 & 10 \\
\hline 6. & 10 & 15 & 3.73 & 49.41 & 3 & 4.8 & 37.8 & 3.4 & 0.336 & 63.4 & 6 \\
\hline 7. & 10 & 20 & 6.64 & 69.46 & 4 & 6.6 & 70.8 & 22.2 & 0.334 & 54.5 & 11 \\
\hline 8. & 10 & 30 & 14.94 & 78.53 & 3 & 7.0 & 158.9 & 50.6 & 0.263 & 55.1 & 9 \\
\hline 9. & 15 & 10 & 1.66 & 12.64 & 3 & 2.7 & 36.8 & 2.3 & 0.543 & 58.2 & 12 \\
\hline 10. & 15 & 15 & 3.73 & 46.91 & 3 & 4.0 & 35.8 & 2.5 & 0.323 & 66.1 & 7 \\
\hline 11. & 15 & 20 & 6.64 & 84.09 & 3 & 2.2 & 129.3 & 6.5 & 0.682 & 62.5 & 10 \\
\hline 12. & 15 & 30 & 14.94 & 89.93 & 3 & 6.4 & 153.9 & 32.9 & 0.264 & 55.4 & 9 \\
\hline 13. & 20 & 20 & 6.64 & 71.44 & 3 & 3.8 & 127.3 & 8.7 & 0.534 & 58.4 & 8 \\
\hline 14. & 20 & 30 & 14.94 & 86.36 & 3 & 5.9 & 275.8 & 22.2 & 0.427 & 59.8 & 7 \\
\hline 15. & 25 & 20 & 6.64 & 80.81 & 3 & 1.5 & 129.1 & 7.8 & 0.735 & 64.4 & 4 \\
\hline 16. & 25 & 30 & 14.94 & 101.15 & 5 & 5.3 & 275.7 & 22.7 & 0.514 & 58.3 & 6 \\
\hline
\end{tabular}

The data files were further processed with the program compiled in MathCad soft to reduce signal noise and obtain curves of discharge parameters: current $I(t)$, voltage $V(t)$, power $p(t)$, energy $E(t)$. Time parameters were determined from the graphs and written in Table 1: quantity of semi-periods of discharge current $n$, duration of the first semi-period of discharge current $\tau_{1}$.

\section{Tests results and data processing}

The values of dimples heights $h_{i}$ were measured and pressure values $P_{i}$ were calculated for each $i$ point of a membrane after impact loading at selected test conditions. Then the following parameters were calculated:

- $P_{\max }$-maximum pressure selected from a pressure map (from $P_{i}$ array) and assumed as a peak pressure of shock wave $P_{m}=P_{\text {maxi }}$

- Average peak pressure calculated from the $k$ tests produced under the same conditions

$$
P_{m-a v e}=\frac{1}{k} \sum_{i=1}^{k} P_{m i} .
$$

In this work those pressure tests results submitted by San José et al. [3] are used for further analysis (Table 1). Quantity of tests for pressure parameters in definite series is $k$.

Measurements of electric discharge parameters were produced under the same conditions applied for the same

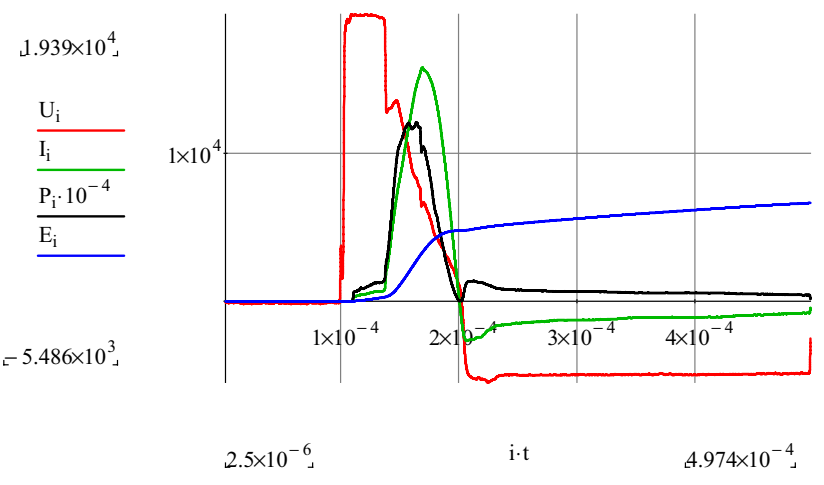

Fig. 8 Representative discharge curves of voltage Vi, current li, power $\mathrm{Pi}$ and evolved energy Ei processed in MathCAD for the test No. 15-3 with initial electric parameters: charge voltage $V 0=20 \mathrm{kV}$, capacitance $C=33.2 \mu \mathrm{F}$, charge energy $E O=6.64 \mathrm{~kJ}$, spark gap $I=25 \mathrm{~mm}$. Time $t$ scale is in seconds

series of pressure measurements, including spark gap distance $I$, charge voltage $V_{0}$, capacitance $C$, charged energy $E_{0}$, distance between spark gap and membrane pressure gauge $H$. Quantity of tests for energy parameters calculation is $m$ in definite series.

Energy parameters of discharges were calculated from electric parameters voltage $V(t)$ and current $I(t)$ values (curves): electric power $N(t)=V(t) \cdot I(t)$, energy values $E(t)=\Sigma(N(t) \cdot \Delta t)($ Fig. 8).

In the Table 1 the average values of electric parameters were calculated from the following formulas: 
- Average quantity of semi-periods of discharge current for $m$ tests with the same conditions

$$
n_{\text {ave }}=\frac{1}{m} \sum_{i=1}^{m} n_{i}
$$

- Average maximum value of power during the first semi-period of discharge current

$$
n_{\max -a v e}=\frac{1}{m} \sum_{i=1}^{m} N_{i \max }
$$

- Average duration of the first semi-period of discharge current

$$
\tau_{\text {1ave }}=\frac{1}{m} \sum_{i=1}^{m} \tau_{i}
$$

- Average quantity of energy evolved during the first semi-period of discharge current

$$
E_{\text {1ave }}=\frac{1}{m} \sum_{i=1}^{m} E_{1 i} ;
$$

- Average ratio of energy evolved during the first semiperiod of discharge current

$$
\eta_{\text {ave }}=\frac{1}{m} \sum_{i=1}^{m} \eta_{i} ;
$$

\section{Analysis of tests results}

For better understanding of experimental data and their analysis they are represented in the form of graphs.

General characteristic of many discharges in relation to the peak pressure of shock wave $P_{m \text {-ave }}$ and spark gap value

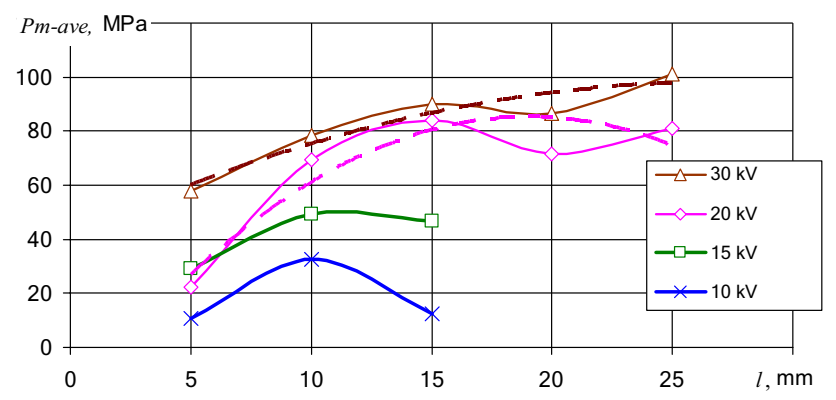

Fig. 9 Experimental curves $P m$-ave(I) of peak pressure plotted from average values of tests for different charge voltages. Approximation curves of $20 \mathrm{kV}$ and $30 \mathrm{kV}$ are shown by dotted lines
I is submitted in Fig. 9. These curves are plotted from the average values of Table 1.

The first analysis in comparison with the Krivitsky and Shamko [6] data (Fig. 6) shows that curves of $10 \mathrm{kV}$ and $15 \mathrm{kV}$ confirm the general law of optimal spark gap value for the given electric parameters of discharge circuit. And this optimal gap value corresponds to the practical rule of $1 \mathrm{~mm}$ per $1 \mathrm{kV}$ applied for industrial EHF installations.

Approximation curves of $20 \mathrm{kV}$ and $30 \mathrm{kV}$ shown by dotted lines in Fig. 9 also agree with general rule for the optimal spark gap value (Fig. 6). The approximation curve of $20 \mathrm{kV}$ has maximum near to $20-\mathrm{mm}$ gap value, and approximation dotted curve of $30 \mathrm{kV}$ does not reach its extremum, because of the $25-\mathrm{mm}$ spark gap limit in this series of tests.

But both spline curves have depressions (concavities) corresponding to spark gap value $I=20 \mathrm{~mm}$. And these facts fall out the smooth approximation curves in Fig. 9 and the curve in Fig. 6 that contradicts the general law of optimal spark gap value. These deviations in peak pressure can reach $20-30 \%$.

This situation needs more detailed analysis with consideration of other discharge energy parameters: maximum power $N_{\max }$ and slope of power curve $\gamma_{1}$ proposed by Krivitsky and Shamko [6].

The experimental curves $N_{\text {max }}(l)$ depicted in Fig. 10 have rising nature in the specified range of spark gap values and criteria $\eta$ (ref. Table 1 ) and do not reach the maximum values to be at $\eta=0.7-0.8$.

Figure 10 confirms, in general, that the larger voltage and respectively higher energy are realised in increase of maximum power during the first semi-period of the discharge current that corresponds to the maximum pressure curves in Fig. 9 and the Krivitsky and Shamko [6] conclusions. In general curves of 20 and $30 \mathrm{kV}$ also agree to previous results submitted in Fig. 3, considering the criteria $\eta$ increases with spark gap enlargement till some limit. But there are several deviations.

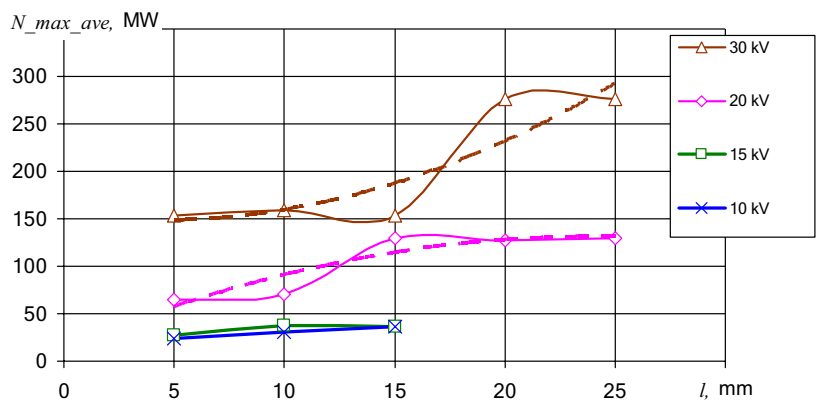

Fig. 10 Experimental curves Nmax-ave(l) maximum power during the first semi-period of the discharge current plotted from average values of tests for different charge voltages 


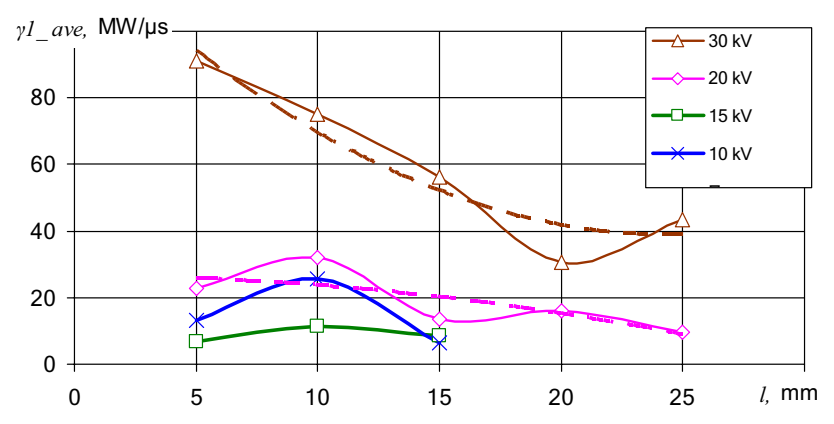

Fig. 11 Experimental curves $\gamma 1$-ave(l) of power curve slope during the first quarter of discharge current period plotted from average values of tests for different charge voltages

Thus, in Fig. 10 the discharges at lower voltage of $10 \mathrm{kV}$ and $15 \mathrm{kV}$ show approximately the same level of maximum power that contradicts to different values of respective pressure curves in Fig. 9.

The curve of 20-kV discharges shows approximately the same power maximums at different spark gap values of $15 \mathrm{~mm}, 20 \mathrm{~mm}$ and $25 \mathrm{~mm}$. Respective points in Fig. 9 indicate approximately the same peak pressure at $15-\mathrm{mm}$ and 25-mm spark gap, but one can observe pressure drop at the 20-mm spark gap. Also, the 20-kV curve shows 2-time higher power at small gap $5 \mathrm{~mm}$ as compared with $15-\mathrm{kV}$ discharges, but the peak pressure appeared to be lower (Fig. 9).

The curve of 30-kV discharges shows approximately the same power maximum at different spark gap values of 10 , 15 and $20 \mathrm{~mm}$, but respective points in Fig. 9 indicate rise of peak pressure with gap enlargement that corresponds to Fig. 6.

Another parameter influencing value of the peak pressure is a slope of power curve $\gamma_{1}$ in the first quarter of discharge current period. Figure 11 shows that, in general peak, pressure increases with enlargement of maximum power curve slope in dependency of voltage (and energy) of discharge. But slope dependencies of spark gap (and criteria $\eta$ ) are monotonously decreasing curves for the 20-kV and 30-kV discharges and have no extremums as it was informed by Krivitsky and Shamko [6] about change of sign of derivative $\frac{d}{d l} P_{m}(l)$ and respective change of dependencies of power $N_{\max }$ and slope of power curve $\gamma_{1}$ (ref. formula (1)).

The slope of power curves for the "long" discharges $\left(\tau_{1}>40 \mu \mathrm{s}\right)$ should be maximal and nearly the same for wide range of discharge modes determined by the criteria $\eta=0.1-0.7$ (Fig. 3). But experimental curves $\gamma_{1 \text {-ave }}(l)$ demonstrate monotonous decrease in the mentioned range of criteria $\eta$ (Table 1) for the 20-kV and 30-kV discharges.

Another contradiction is in discharges at voltage $10 \mathrm{kV}$ and $15 \mathrm{kV}$. Though the $10-\mathrm{kV}$ curve shows bigger slopes of power curves the peak pressure values $P_{\text {m-ave }}(l)$ are smaller than the pressure values at $15 \mathrm{kV}$ (Fig. 9). At that, both curves have extremums, which correspond to peak pressure extremums.

Here the authors should assume that they cannot exclude significant influence of signal noise on the measuring and processing results, which is rather usual for impulse high-voltage equipment. But these errors cannot be so large to catastrophically changing the essence of obtained results.

Thus, this general analysis along average peak pressure and energy parameters showed that significant deviations from the previous theoretical and laboratory investigation results [5-7] can appear in industrial EHF installations with non-initiated discharges. These deviations can create instability problems in forming of sheet parts. Therefore, the causes of these problems should be revealed and eliminated in order to harmonise generation of pressure pulses and processes of sheet forming.

\section{Discussion}

The general analysis above shows that significant deviations from the previous theoretical and laboratory investigation results can appear in industrial EHF installations with non-initiated discharges. This means that in industrial installations some factors, which were not considered in previous investigations [5-7], appear and influence the pressure parameters of discharges.

These "additional" factors, peculiar to industrial EHF installations, are probable: condition of current-conductive rod of electrode (erosion, rust, radius); condition (wear) of electrode insulator (increase of naked area of current-conductive rod); variations in shape, position and length of discharge channel relative to spark gap; "shadowing" effect of electrodes at some positions of discharge channel relative to electrode; several discharge channels at small spark gap, etc.

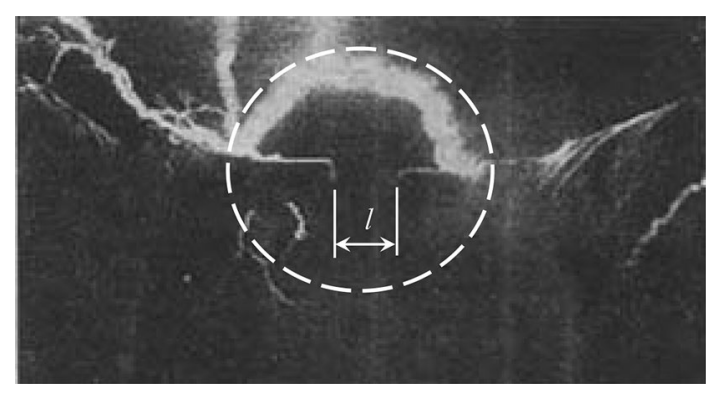

Fig. 12 Electric discharge channel in a liquid [7]. Volume of probable positions of channel is shown by dotted line in comparison with length / of spark gap 


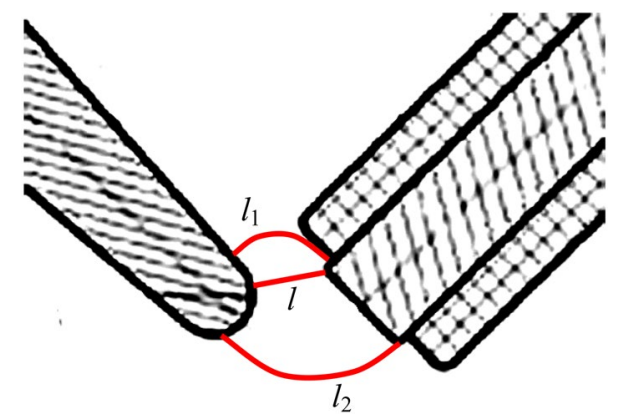

Fig. 13 Variations in actual length of discharge channel in comparison with spark gap distance

The physics of electric discharge in water had been investigated by Naugolnykh and Roy [7]. Figure 12 confirms that actual length of discharge channel can greatly exceed spark gap value and the position of actual channel relative to axis of electrodes is rather probabilistic parameter. As a rule, discharge channel originates from the boundary line between current-conductive rod and insulator of one electrode and completes the discharge circuit also on the boundary line of the opposite electrode (Fig. 12). Thus, discharge channel can appear in any position in the volume with length from one boundary line to another and with radius equals approximately 2 spark gap values (shown by dotted line). This volume can be spherical, when spark gap is small, and ellipsoidal, when spark gap is large. Here we can assume that deviation of discharge channel from the electrodes axis depends on charged voltage and energy in relation to spark gap value and electrodes geometric configuration. Guly [5] and Krivitsky and Shamko [6] also mentioned that large dispersion of pre-discharge time is caused by large naked area of electrodes. Also, for large charged energy at small spark gap several discharge channels can be generated simultaneously. Then they can merge with each other and create single total channel that can influence energy and pressure parameters of discharge.

Based on the discharge photos [7] the diagram of possible channel shapes and positions can be plotted for the series of tests considered in this work. Figure 13 shows the volume of probabilistic positions of discharge channel at some predetermined value of spark gap. Line $I_{1}$ shows supposed extreme upper position and shape of discharge channel and the line $I_{2}$-discharge channel in the lowest extreme position. It is obvious that for noninitiated discharges in industrial chambers the length of discharge channel can vary from the smallest and equal to the spark gap length $I$ to the largest $I_{2}$ length that can 2-3 times exceed the distance l; respectively, active resistance $R$ of discharge channel will vary in the wide range. That, in its turn, will influence all other electric and energy discharge parameters, which in their turn, will influence the peak pressure of generated shock wave and its propagation to a sheet blank. Also, it is quite obvious that at upper position of discharge channel the electrodes will reflect some portion of direct shock wave and create "shadowing" effect that will greatly reduce intensity of shock wave pressure in downwards direction.

\section{Conclusions}

Dependencies of peak pressure $P_{m}$ from maximum power $N_{\text {max }}$ during the first semi-period of discharge current and slope of power curve $\gamma_{1}$ appeared to be not so strong. In the specified range of spark gap values the experimental curves $N_{\max }(I)$ and $\gamma_{1 \text {-ave }}(I)$ do not contain extreme points that would correspond to extreme points of the $P_{m}(I)$ curves at different levels of charged voltage and energy. This situation creates some kind of uncertainty in determination of electric parameters of discharge circuit in order to generate the largest peak pressure of shock wave.

Conducted experimental investigation has shown that industrial applications of high-voltage non-initiated discharges can significantly deviate from the theoretical and laboratory results.

Among the assumed additional factors influencing energy and pressure parameters are: variations in shape, position and length of discharge channel relative to spark gap; "shadowing" effect of electrodes at some positions of discharge channel relative to electrodes and other.

To reveal effect of these factors the authors plan to carry-out tests with wire-initiated discharges to check the variations in shape, position and length of discharge channel relative to spark gap in the same discharge chamber configuration, as well as to investigate other features of electric energy evolvement and peak pressure generation.

\section{Compliance with ethical standards}

Conflict of interest The authors declare that they have no conflict of interest.

\section{References}

1. Vohnout VJ, Fenton G, Daehn GS (2010) Pressure heterogeneity in small displacement electrohydraulic forming processes. In: Proceedings of the 4th international conference on high speed forming, pp 65-74

2. Homberg W, Beerwald C, Probsting A (2010) Investigation of the electrohydraulic forming process with respect to the 
design of sharp-edged contours. In: Proceedings of the 4th international conference on high speed forming, pp 58-64

3. San José J, Perez I, Knyazyev MK, Zhovnovatuk YS (2012) Pressure fields repeatability at electrohydraulic pulse loading in discharge chamber with single electrode pair. In: Proceedings of the 5th international conference on high speed forming (ICHSF), pp 33-42

4. San José J, Perez I, Knyazyev MK, Zhovnovatuk YS (2012) Investigation of repeatability problem in electrohydraulic forming of sheet components. In: Proceedings of the international conference IDDRG2012, pp 333-338

5. Guly GA (1977) Equipment and manufacturing processes with application of electrohydraulic effect. Edited by Mashinostroeniye (in Russian)

6. Krivitsky EV, Shamko VV (1979) Transient processes at high-voltage discharge in water. Naukova Dumka Publishers (in Russian)

7. Naugolnykh KA, Roy NA (1971) Electric discharges in water. Nauka ed (in Russian)

8. Homberg W, Akst O, Djakow E (2012) Some aspects regarding the use of a pneumomechanical high speed forming process. In: Proceedings of the 5th international conference on high speed forming, pp 23-32

9. Mamutov V, Golovashchenko S, Mamutov A (2014) Simulation of high-voltage discharge channel in water at electrohydraulic forming using LS-DYNA ${ }^{\circ}$. In: Proceedings of the 13th international LS-DYNA users conference, pp 1-9

10. Mamutov AV, SF Golovashchenko AV, Mamutov AV, Bonnenc JJF (2015) Modelling of electrohydraulic forming of sheet metal parts. J Mater Process Technol 219:84-100

11. Shim JY, Kang BY (2017) Development of electrohydraulic forming process for aluminum sheet with sharp edge. Adv Mater Sci Eng

12. Coman $L$ (2010) Influence of some factors on maximum depth in electrohydraulic forming. Analele Universitatii "Eftimie Murgu" Resita, Anul XVII, NR. 2, pp 93-98

13. Knyazyev MK, Zhovnovatuk YS (2010) Measurements of pressure fields with multi-point membrane gauges at electrohydraulic forming. In: Proceedings of the 4th international conference on high speed forming, pp 75-82

14. Antonenko AA et al (2004) Investigation of electrohydraulic forming processes in installations with multi-electrode discharge blocks. East Eur J Enterp Technol 1(7):23-32 (in Russian)

Publisher's Note Springer Nature remains neutral with regard to jurisdictional claims in published maps and institutional affiliations. 